\title{
Characterization of Polarons in Armchair Graphene Nanoribbons
}

Marcelo Macedo Fischer ${ }^{1}(P G)$, Leonardo Luiz e Castro $^{2}$ (PQ), Pedro Henrique de Oliveira $\mathrm{Neto}^{3}$ (PQ)

${ }^{1}$ Universidade de Brasília, 170175600@aluno.unb.br, ${ }^{2}$ Universidade de Brasília, llcastro@fis.unb.br, ${ }^{3}$ Universidade de Brasília, pedrohenrique@unb.br

Keywords: Polarons, graphene nanoribbons, charge carrier mobility, AGNR

\section{Introduction}

Graphene is a 2D material with several important applications mainly because of its resistance against compression and traction, low cost, high flexibility, among other properties. Also, graphene is transparent to UV radiation, visible light, and infrared light, containing an interesting charge transport mechanism. It's stable at ambient temperature and is a great conductor of heat and electric current. As graphene nanoribbons (GNRs), the system may present intrinsic bandgap and high charge carriers mobility, being a potential material for optoelectronics application.

The electronic states of GNRs depend on the edge structures, where the main ones are armchair and zigzag configurations. In the systems presenting semi-metallic behavior, the charge carriers are quasiparticles with strong electron-phonon coupling known as polarons[1]. In this work, we investigate the polarons mobility in armchair GNRs (AGNRs) with several widths. In a phenomenological approach, we characterize the systems by a kinematic study.

\section{Methodology}

The graphene lattice is described by a tight binding Hamiltonian model that takes into account the electron phonon-coupling, and an external electric field. Methodologically, in the Self Consistent Field approach, we used the Ehrenfest molecular dynamic to study the polaron motion under the influence of an external electric field. In each time step, we evaluate the charge density of the system. Then, one can calculate the center of charge and observe how the polaron evolves on time. The present study was performed for a range of constant external electric fields for systems varying from 4 to 9 Carbons in width named here as 4-AGNR to 9-AGNR.

\section{Results}




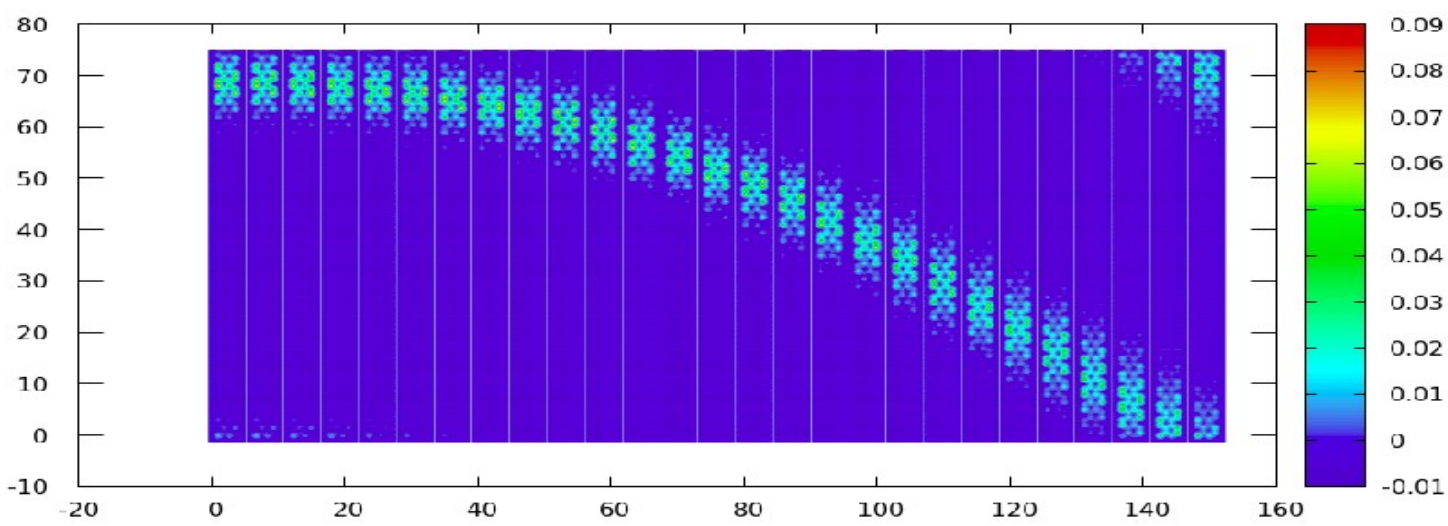

Image 1: A single polaron moving on a 4-AGNR, under influence of a constant external electric field for $2 p s$.

Figure 1 shows the charge density time evolution of a polaron moving under the influence of a constant external electric field, that is applied in the vertical direction, for the 4-AGNR. The vertical axis is the nanoribbon's length and the horizontal axis is the time in fs. A periodic boundary condition was applied in the vertical direction in order to avoid edge effects. It is possible to note that the charge is accelerated in the beginning and then reaches a terminal velocity, roughly by half of the simulation.

\section{Discussion}

The polaron motion follows a Newtonian-type equation described by a constant force with a Stokes dissipation term. By modeling the kinematics, we evaluate the effective mass and the drag coefficient. The terminal velocity gives the drag coefficient while the fitting by the solution of the equation of motion gives the effective mass.

\section{Conclusions}

In conclusion, using a Hamiltonian model in the Ehrenfest molecular dynamics we studied the motion of the charge carriers in armchair graphene nanoribbons. By modeling its kinematics, we have characterized polarons in terms of its effective mass and drag coefficient.

\section{Acknowledgment}

The authors gratefully acknowledge the financial support from CAPES, CNPq and FAP-DF.

[1] DE OLIVEIRA NETO, PEDRO HENRIQUE; VAN VOORHIS, TROY . Dynamics of charge quasiparticles generation in armchair graphene nanoribbons. CARBON ıcR, v. 132, p. 352-358, 2018. 